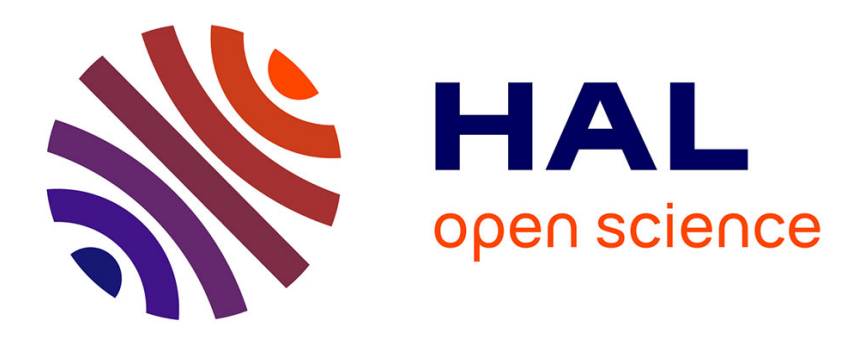

\title{
A Local Surface Model applied to Contact Line Dynamics
}

\author{
Jerome Monnier, Patrick Witomski
}

\section{To cite this version:}

Jerome Monnier, Patrick Witomski. A Local Surface Model applied to Contact Line Dynamics. Nonlinear Analysis: Theory, Methods and Applications, 2005, 63 (5-7), pp.1551-1559. 10.1016/j.na.2005.01.049 . inria-00256607

\section{HAL Id: inria-00256607 https://hal.inria.fr/inria-00256607}

Submitted on 15 Feb 2008

HAL is a multi-disciplinary open access archive for the deposit and dissemination of scientific research documents, whether they are published or not. The documents may come from teaching and research institutions in France or abroad, or from public or private research centers.
L'archive ouverte pluridisciplinaire HAL, est destinée au dépôt et à la diffusion de documents scientifiques de niveau recherche, publiés ou non, émanant des établissements d'enseignement et de recherche français ou étrangers, des laboratoires publics ou privés. 


\section{ARTICLE IN PRESS}

Available online at www.sciencedirect.com

science d Direct.

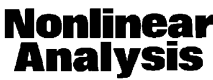

ELSEVIER

\section{A local surface model applied to contact line dynamics}

\section{J. Monnier*, P. Witomski}

Projet IDOPT (CNRS-INPG-INRIA-UJF), Laboratoire de Modelisation et Calcul (LMC-IMAG), BP 53, F-38041 Grenoble Cedex 9, France

\section{Abstract}

We consider a set of equations modeling contact line dynamics. The model consists of the

9 Navier-Stokes free surface flow with local slip-type boundary conditions and gradient surface tension coupled with a mesoscopic local surface model (nonlinear degenerated equations) describes the surface tension variations. The dynamical contact angle and the local surface tension variations are unknowns of the model. We present some mathematical and numerical results.

\section{Introduction}

The aim of the paper is the mathematical and numerical modeling of dynamical contact 17 lines (e.g. coating of solids by liquids). Two main features of such flows are the following. First, the liquid front advances following a rolling motion, similar to a caterpillar vehicle, see [3]. Second, the dynamical contact angle deviates from its static value, determined by the classical Young equation, and depends on the fluid velocity in the bulk. In addition, it seems that its value cannot be prescribed explicitly in a general way, see e.g. [1]. The mathematical modeling of the moving contact line is delicate. A no-slip boundary condition at the solid-liquid interface implies a non-physical singularity: the fluid exerts an infinite

\footnotetext{
* Corresponding author.

E-mail address: jerome.monnier@imag.fr (J. Monnier).
} 
1 force on the solid surface [3]. Then, most of the theories and most of models have been based on a slippage description, see e.g. [6,2,4,7].

3 The mathematical model studied in the present paper is based on the model established in [7,1]. The main idea of this model is to take into account the rolling motion

5 induces a local variation of the surface tension [7]. The induced surface tension gradient influences the motion and the force near the contact line; it implies a Marangoni ef-

7 fect. In this model, the (dynamical) wetting angle is not imposed but is a response of the model.

\section{2. The mathematical model}

In this section, we consider the configuration of a solid plate plunging vertically into a 2D pool of liquid at speed $U_{\mathrm{S}}$, Fig. 2, and we present a model derived from [7].

We denote by $\Omega$ the liquid pool wetting the solid tape $(S)$, by $\Gamma_{\mathrm{SL}}$ the solid-liquid contact surface, by $\Gamma_{\mathrm{SG}}$ the solid-gas contact surface, by $\Gamma_{\mathrm{LG}}$ the free surface liquid-gas and by $P_{\mathrm{C}}$ the contact point liquid-gas-solid.

15 When the liquid is at rest, the (static) contact angle $\theta_{\mathrm{S}}$ satisfies the classical Young equation: $\sigma_{\mathrm{LG}}^{\mathrm{eq}} \cos \left(\theta_{\mathrm{S}}\right)=\sigma_{\mathrm{SG}}^{\mathrm{eq}}-\sigma_{\mathrm{SL}}^{\mathrm{eq}}$, where $\sigma_{\mathrm{LG}}^{\mathrm{eq}}, \sigma_{\mathrm{SL}}^{\mathrm{eq}}$ and $\sigma_{\mathrm{SG}}^{\mathrm{eq}}$ are the equilibrium surface tensions 17 of the liquid-gas, solid-liquid and solid-gas interfaces, respectively. In this paper, we consider the dynamic case where the solid plate is moving at speed $U_{\mathrm{S}}$, see Fig. 2 . In that case, the contact angle becomes variable. The basic idea of the model studied in [7] is to consider that the Young equation remains valid:

$$
\sigma_{\mathrm{LG}} \cos \left(\theta_{\mathrm{d}}\right)=\sigma_{\mathrm{SG}}-\sigma_{\mathrm{SL}}
$$

where $\theta_{\mathrm{d}}$ denotes the dynamic contact angle.

Briefly, the full model considered in the present paper is as follows. A macroscopic hydrodynamic free surface model, HFSM, for the fluid motion is coupled to a mesoscopic local surface model, LSM, describing the local surface tension distribution and the contact line motion. The HFSM consists of the Navier-Stokes equations with free surface and slip-type boundary conditions. The coupling with the LSM is done through these boundary conditions imposed on a small vicinity of the triple line. The LSM describes the dependence between the surface tension parameters and the fluid motion.

\subsection{The macroscopic hydrodynamic free surface model}

We denote by $\vec{u}$ the fluid velocity, $p$ its pressure, $\Sigma$ the stress tensor with components $\Sigma_{i j}=-p \delta_{i j}+\mu\left(\partial_{i} u_{j}+\partial_{j} u_{i}\right) 1 \leqslant i, j \leqslant 2$, where $\mu$ is the dynamic viscosity.

We denote by $(\vec{\tau}, \vec{n})$ the unit tangential and external normal vectors such that it is direct. We set: $\vec{\Sigma}_{n}=\Sigma^{\prime} \cdot \vec{n} \in \mathbb{R}^{2} ; \vec{\Sigma}_{n}=\Sigma_{n} \vec{n}+\Sigma_{\tau} \vec{\tau}$.

The fluid motion is governed by the incompressible Navier-Stokes equations (into variables $(\vec{u}, p))$. To describe the boundary conditions, we decompose $\Gamma_{\mathrm{SL}}$ (respectively, $\Gamma_{\mathrm{LG}}$ ) in two parts $\Gamma_{\mathrm{SL}}^{\mathrm{M}}$ and $\Gamma_{\mathrm{SL}}^{\mathrm{m}}$ (respectively, $\Gamma_{\mathrm{LG}}^{\mathrm{M}}$ and $\Gamma_{\mathrm{LG}}^{\mathrm{m}}$ ). The superscripts ${ }^{\mathrm{M}}$ and ${ }^{\mathrm{m}}$ refer to 
1 the macroscopic and the mesoscopic boundaries, respectively. The boundary conditions on the free surface (liquid-gas) are

$$
\begin{cases}\vec{\Sigma}_{n}=\left(-p_{\mathrm{ext}}+\sigma_{\mathrm{LG}} \kappa\right) \vec{n} & \text { in }(0, T) \times \Gamma_{\mathrm{LG}}^{\mathrm{M}}, \\ \vec{\Sigma}_{n}=\left(-p_{\mathrm{ext}}+\sigma_{\mathrm{LG}} \kappa\right) \vec{n}+\left(\vec{\nabla} \sigma_{\mathrm{LG}} \cdot \vec{\tau}\right) \vec{\tau} & \text { in }(0, T) \times \Gamma_{\mathrm{LG}}^{\mathrm{m}},\end{cases}
$$

where $\kappa$ is the mean curvature and $p_{\text {ext }}$ is the external pressure.

5 The liquid-solid contact is described by: $\vec{u}=\vec{U}_{\mathrm{S}}$ in $(0, T) \times \Gamma_{\mathrm{SL}}^{\mathrm{M}}$, where $\vec{U}_{\mathrm{S}}$ is the solid velocity, and

$$
\begin{cases}\vec{u} \cdot \vec{n}=0 & \text { in }(0, T) \times \Gamma_{\mathrm{SL}}^{\mathrm{m}}, \\ \sum_{\tau}=-\left(\beta\left(\vec{u}-\vec{U}_{\mathrm{S}}\right)-\frac{1}{2} \vec{\nabla} \sigma_{\mathrm{SL}}\right) \cdot \vec{\tau} & \text { in }(0, T) \times \Gamma_{\mathrm{SL}}^{\mathrm{m}},\end{cases}
$$

where $\beta>0$ is a sliding-type coefficient.

The boundary condition (2) removes the shear-stress singularity. Surface tension gradients appear in (1) and (2). It is one of the novel features of the model.

We define the free surface $\Gamma_{\mathrm{LG}}$ as the graph of a function $\varphi(t, x)$ and the free surface motion is described by the classical transport equation with the graph value given at the inflow boundary.

Remark 2.1. Let us point out an important feature of the model. The dynamic wetting angle $\theta_{\mathrm{d}}$ is not imposed. It is a response of the model. It can be computed using the relation: $\operatorname{cotan}\left(\theta_{\mathrm{d}}\right)=-\left(\partial \varphi / \partial x_{1}\right)\left(t, P_{\mathrm{C}}\right)$ for $t$ in $(0, T)$.

\subsection{The mesoscopic local surface model}

Briefly, the so-called mesoscopic LSM (established in [7]) is as follows. The interfaces are described by surface densities $\rho^{\mathrm{s}}$ which are solution of surface continuity equations. A state equation provides the relation between $\rho^{\mathrm{s}}$ and the surface tension coefficients $\sigma$. We denote by $\rho_{i}^{\mathrm{s}}, \quad i=1,2$, the surface density on $\Gamma_{\mathrm{LG}}(i=1)$ and on $\Gamma_{\mathrm{SL}}(i=2)$. The surface tension is related to the excess density through a linear state equation

$$
\sigma_{i}=\gamma\left(\rho_{0}^{\mathrm{s}}-\rho_{i}^{\mathrm{s}}\right), \quad i=1,2,
$$

where $\gamma$ and $\rho_{0}^{\mathrm{s}}$ are given constants. We have the surface continuity equation

$$
\frac{\partial \rho_{i}^{\mathrm{s}}}{\partial t}+\operatorname{div}\left(\rho_{i}^{\mathrm{s}} v_{i}^{\mathrm{s}}\right)+\frac{1}{\tau^{*}}\left(\rho_{i}^{\mathrm{s}}-\rho_{i}^{\mathrm{eq}}\right)=0, \quad i=1,2,
$$

where $\tau^{*}$ is the relaxation time relative to the rolling motion, $v_{i}^{\mathrm{s}}$ is a mean velocity inside the layer and $\rho_{i}^{\mathrm{eq}}$ is its density at equilibrium: $\sigma_{i}\left(\rho_{i}^{\mathrm{eq}}\right)=\sigma_{i}^{\mathrm{eq}}, i=1,2$.

The velocity $v_{1}^{\mathrm{s}}$ (respectively, $v_{2}^{\mathrm{s}}$ ) is related to $\rho_{1}^{\mathrm{s}}$ (respectively, $\rho_{2}^{\mathrm{s}}$ ) and to the fluid velocity $u$ (respectively, the solid velocity $U_{\mathrm{S}}$ ). We have the following Darcy laws type:

$$
\left(1+4 \alpha_{1} \alpha_{2}\right) \nabla \sigma_{\mathrm{LG}}=4 \alpha_{2}\left(v_{1}^{\mathrm{s}}-u\right) \quad \text { and } \quad v_{2}^{\mathrm{s}}=\alpha_{1} \nabla \sigma_{\mathrm{SL}}+\frac{1}{2}\left(u+U_{\mathrm{S}}\right),
$$

31 where $\alpha_{i}, i=1,2$, are given constants characterizing the viscous properties of the interface. At the triple junction, the surface flux continuity is imposed: $\left(\rho_{1}^{\mathrm{s}} v_{1}^{\mathrm{s}}\right) e_{\mathrm{f}}=\left(\rho_{2}^{\mathrm{s}} v_{2}^{\mathrm{s}}\right) e_{\mathrm{g}}$, where 
$1 e_{\mathrm{f}}$ and $e_{\mathrm{g}}$ are unit vectors normal to the contact line and tangential to the gas-liquid and gas-solid interface, respectively. Let us notice that $\cos \left(\theta_{\mathrm{d}}\right)=-e_{\mathrm{f}} \cdot e_{\mathrm{g}}$.

\section{3. The $1 \mathrm{D}$ local surface model}

We consider the 1D steady-state LSM. We reformulate the equations by eliminating the 5 variable $v_{i}^{\mathrm{s}}$. For both cases $i=1$ and 2 , we obtain similar equations. They are nonlinear and degenerated. Case $i=2$ (solid-liquid surface) leads to:

7

(P) $\left\{\begin{array}{l}\left.-\left(\rho \rho^{\prime}\right)^{\prime}+\delta_{1} U \rho^{\prime}+\delta_{2} \rho=f \text { in }\right] 0,1[, \\ \rho(0)=\rho_{0}, \\ \left(-\rho \rho^{\prime}+\delta_{1} U \rho\right)(1)=\phi,\end{array}\right.$

where $\delta_{1}=l U^{*} / \lambda \rho^{*}, \delta_{2}=l^{2} / \lambda \rho^{*} \tau^{*}$ are dimensionless numbers, $\phi=\delta_{1} \rho_{1}^{\mathrm{eq}}(2 U(1)-$

$9 U(0)) \leqslant 0, \phi$ is the flux at the contact point and $f=\delta_{2} \rho_{0}$. Let us notice that if we set $l=\tau^{*} U^{*}$, then $\delta_{1}=\delta_{2}=\frac{\tau^{*}\left(U^{*}\right)^{2}}{\lambda \rho^{*}}$.

\section{3.1. Mathematical analysis}

Let us assume

\section{Assumption 3.1.}

(i) $\rho_{0}>0$.

15 (ii) $U \in W^{1, \infty}(0,1)$ and $U \leqslant 0$ in $[0,1] ; U^{\prime} \geqslant 0$ a.e. and $\left\|U^{\prime}\right\|_{\infty} \leqslant \delta_{2} / \delta_{1}$.

We consider the nonlinear regularized problem:

$$
\left(\mathrm{P}^{\beta}\right) \quad\left\{\begin{array}{l}
\left.-\left(\beta_{\varepsilon}(\rho) \rho^{\prime}\right)^{\prime}+\delta_{1} U \rho^{\prime}+\delta_{2} \rho=f \quad \text { in }\right] 0,1[, \\
\rho(0)=\rho_{0}, \\
\left(-\beta_{\varepsilon}(\rho) \rho^{\prime}+\delta_{1} U \rho\right)(1)=\phi,
\end{array}\right.
$$

where $\varepsilon>0, \beta_{\varepsilon} \in C^{1}(\mathbb{R})$ is Lipschitz, increasing and defined by: $\beta_{\varepsilon}(x)=\varepsilon$ if $x \leqslant 0$ and $19 \beta_{\varepsilon}(x)=x$ if $x \geqslant 2 \varepsilon$.

Using the Leray-Schauder fixed-point theorem, we prove that under Assumption 3.1,

21 Problem $\left(\mathrm{P}^{\beta}\right)$ has at least one weak solution in $H^{1}(0,1)$ and this solution belongs to $H^{2}(0,1)$.

23 Furthermore, let $\eta$ be a real number satisfying: $\rho_{0} \geqslant \eta>0$ and $\eta \delta_{1} U(1) \geqslant \phi$. For example with $U(1)<0$, we may set $\eta=\min \left\{\rho_{0},\left[\phi / \delta_{1} U(1)\right]\right\}$. Combining the weak maximum principle, see [5], and the previous existence result, we obtain:

Theorem 3.1. Under Assumption 3.1, Problem (P) has at least one weak solution $\rho$ in

$27 H^{1}(0,1)$. This solution satisfies $\rho(x) \geqslant \eta>0$ in $[0,1]$ and belongs to $H^{2}(0,1)$.

Actually, we have the following stronger result. 
1 Theorem 3.2. Under Assumption 3.1, Problem (P) has an unique solution $\rho$.

Proof. We denote by $\rho_{1}$ and $\rho_{2}$ two solutions of (P).

(a) First, we prove that $\rho_{1}^{\prime}(0)=\rho_{2}^{\prime}(0)$. Let us suppose that $\rho_{1}^{\prime}(0)>\rho_{2}^{\prime}(0)$. Let $\left.] 0, \xi_{0}\right]$ the largest interval such that $\left.\rho_{1}(x)>\rho_{2}(x), x \in\right] 0, \xi_{0}[$.

5 Let us suppose $\xi_{0}=1$. We integrate the first equation of $(\mathrm{P})$ on $[0,1]$ with $\rho_{1}$ and $\rho_{2}$. By differentiating we obtain:

$$
\begin{aligned}
& -\rho_{1} \rho_{1}^{\prime}(1)+\rho_{2} \rho_{2}^{\prime}(1)+\rho_{0}\left(\rho_{1}^{\prime}(0)-\rho_{2}^{\prime}(0)\right)+\delta_{1}\left[U\left(\rho_{1}-\rho_{2}\right)\right]_{0}^{1} \\
& +\int_{0}^{1}\left(\delta_{2}-\delta_{1} U^{\prime}\right)\left(\rho_{1}-\rho_{2}\right) \mathrm{d} x=0 .
\end{aligned}
$$

Using the boundary conditions of $(\mathrm{P})$, we obtain $\int_{0}^{1}\left(\delta_{2}-\delta_{1} U^{\prime}\right)\left(\rho_{1}-\rho_{2}\right) \mathrm{d} x<0$, which is impossible.

Therefore $\left.\xi_{0} \in\right] 0,1\left[\right.$ and $\rho_{1}\left(\xi_{0}\right)=\rho_{2}\left(\xi_{0}\right)$. We integrate again on $\left[0, \xi_{0}\right]$ and we obtain:

$$
\begin{gathered}
-\rho_{1}\left(\xi_{0}\right)\left(\rho_{1}^{\prime}\left(\xi_{0}\right)-\rho_{2}^{\prime}\left(\xi_{0}\right)\right)+\rho_{0}\left(\rho_{1}^{\prime}(0)-\rho_{2}^{\prime}(0)\right) \\
+\int_{0}^{1}\left(\delta_{2}-\delta_{1} U^{\prime}\right) \cdot\left(\rho_{1}-\rho_{2}\right) \mathrm{d} x=0
\end{gathered}
$$

hence $\left(\rho_{1}-\rho_{2}\right)^{\prime}\left(\xi_{0}\right)>0$. It is impossible, and therefore $\rho_{1}^{\prime}(0)=\rho_{2}^{\prime}(0)$.

(b) Second, we write the first equation of $(\mathrm{P})$ as a first order differential equation of the form: $\left.W^{\prime}(x)=G(W)(x), x \in\right] 0,1\left[\right.$ with $W=(u, v)^{\mathrm{T}}$ and

$$
G(W)(s)=\left(v(s), \frac{-1}{u(s)}\left[-v^{2}(s)+\delta_{1} U(s) v(s)+\delta_{2} u(s)-f(s)\right]\right)^{\mathrm{T}} .
$$

We consider $G: C^{1}([0,1] ; \mathbb{R}) \cap \mathscr{F}^{+} \times C^{0}([0,1] ; \mathbb{R}) \rightarrow C^{0}([0,1] ; \mathbb{R}) \times C^{0}([0,1] ; \mathbb{R})$ with $\mathscr{F}^{+}=\left\{u, u \in C^{0}([0,1] ; \mathbb{R}), u>0\right.$ in $\left.[0,1]\right\}$.

Then, we set $W_{i}=\left(\rho_{i}, \rho_{i}^{\prime}\right)^{\mathrm{T}}, i=1,2$. We have $\left.W_{i}^{\prime}(x)=G\left(W_{i}\right)(x), x \in\right] 0,1[$, and $W_{1}(0)=W_{2}(0)$. Hence, $\left(W_{1}-W_{2}\right)(x)=\int_{0}^{x}\left(G\left(W_{1}\right)-G\left(W_{2}\right)\right)(s) \mathrm{d} s$.

Since $\rho_{i} \in C^{1}([0,1] ; \mathbb{R}) \cap \mathscr{F}^{+}$and $G\left(W_{i}\right)$ is of class $C^{1}$, there exists a constant $k$ such that: $\left\|\left(G\left(W_{1}\right)-G\left(W_{2}\right)\right)(s)\right\| \leqslant k\left\|\left(W_{1}-W_{2}\right)(s)\right\|$.

Then, it follows from the previous equality that: $\left\|\left(W_{1}-W_{2}\right)(x)\right\| \leqslant k \int_{0}^{x} \|\left(W_{1}-W_{2}\right)$ $(s) \| \mathrm{d} s$. And it follows from Gronwall inequality that $W_{1}=W_{2}$ in $[0,1]$.

Proposition 3.1. Let Assumption 3.1 be satisfied and $U$ be such that $U(0)>2 U(1)$.

(i) If $\phi=\delta_{1} U(1) \rho_{0}$ then $\rho \equiv \rho_{0}$, $\rho$ being the unique solution of $(\mathrm{P})$.

(ii) If $\phi<\delta_{1} U(1) \rho_{0}$ then $\rho(x) \geqslant \rho_{0}$ and $\rho^{\prime}(x) \geqslant 0$ in $[0,1]$.

(iii) If $\phi>\delta_{1} U(1) \rho_{0}$ then $\rho(x) \leqslant \rho_{0}$ and $\rho^{\prime}(x) \leqslant 0$ in $[0,1]$.

Proof. (i) It is straightforward to verify that $\rho \equiv \rho_{0}$ is a solution and the solution is unique.

(ii) We have $\phi<\delta_{1} U(1) \rho_{0}$. Since the weak maximum principle holds, we have $\rho \geqslant \rho_{0}$ in $[0,1]$. 
1 Let us prove that $\rho^{\prime}(x) \geqslant 0$. If there exists $\left.\xi_{0} \in\right] 0,1\left[\right.$ such that $\rho^{\prime}\left(\xi_{0}\right)<0$, then

$$
-\left(\rho \rho^{\prime}\right)^{\prime}\left(\xi_{0}\right)+\delta_{1} U\left(\xi_{0}\right) \rho^{\prime}\left(\xi_{0}\right)=\delta_{2}\left(\rho_{0}-\rho\left(\xi_{0}\right)\right) \leqslant 0
$$

3 hence $-\left(\rho \rho^{\prime}\right)^{\prime}\left(\xi_{0}\right)=-\frac{1}{2}\left(\rho^{2}\right)^{\prime}\left(\xi_{0}\right)<0$. We deduce that $\left(\rho^{2}\right)^{\prime}(\xi)>0$ in a neighborhood $\mathscr{V}\left(\xi_{0}\right)$. Therefore $\rho$ is increasing in $\mathscr{V}\left(\xi_{0}\right)$ and $\rho^{\prime}(\xi) \geqslant 0$ in $\mathscr{V}\left(\xi_{0}\right)$, which is a contradiction

5 with $\rho^{\prime}\left(\xi_{0}\right)<0$. Then, we deduce that $\rho^{\prime}(x) \geqslant 0$ in $] 0,1[$ hence in $[0,1]$ since it is continue.

(iii) We prove the result following the same idea as (ii).

\subsection{Numerical results}

We compute numerically the solution of the LSM using a finite difference method. We assume that $\sigma_{\mathrm{LG}}=\sigma_{\mathrm{LG}}^{\mathrm{eq}}$. It follows from (5) that $v_{1}^{\mathrm{s}}=u$ on $\Gamma_{\mathrm{LG}}$. Then, the LSM is reduced to a $1 \mathrm{D}$ differential equation in an interval of the $y$-axis (on $\Gamma_{\mathrm{SL}}^{\mathrm{m}}$ ).

The computation of the 1D mesoscopic LSM provides a profile of $\nabla \sigma_{2}$. In next section this term will be considered as the local Marangoni source term in the Navier-Stokes boundary conditions HFSM.

We consider an air-water-glass system: $\sigma_{L G}^{\mathrm{eq}}=70, \sigma_{\mathrm{SL}}^{\mathrm{eq}}=20$ and $\sigma_{\mathrm{SG}}^{\mathrm{eq}}=50 \mathrm{mN} / \mathrm{m}$.

In the static case, we have: $\cos \left(\theta_{\mathrm{s}}\right)=\left(\sigma_{\mathrm{SG}}^{\mathrm{eq}}-\sigma_{\mathrm{SL}}^{\mathrm{eq}}\right) / \sigma_{\mathrm{LG}}^{\mathrm{eq}} \approx 0.429$ hence $\theta_{\mathrm{s}} \approx 64.6^{\circ}$. In the dynamic case, the Young equation is supposed to remain valid and the case $\theta_{\mathrm{d}}>90^{\circ}$ corresponds to: $\sigma_{\mathrm{SG}}^{\mathrm{eq}}=50<\sigma_{\mathrm{SL}}<\sigma_{\mathrm{LG}}^{\mathrm{eq}}=70$. We set $\tau^{*}=10^{-3} \mathrm{~s}$, see [1], and $U^{*}=5 \times$ $10^{-2} \mathrm{~ms}^{-1}$. Hence, $l \approx \tau^{*} U^{*}=5 \times 10^{-5} \mathrm{~m}$.

We set $U_{\mathrm{S}}=-1=U_{\text {stokes }}(0)$ (the no-slip boundary condition for the bulk flow) and $U(x)=\frac{1}{2}\left(U_{\mathrm{S}}+U_{\text {stokes }}(x)\right)=\left(\frac{1}{4} x-1\right)$.

21 It remains to set the following two parameters: the product $\lambda . \rho^{*}$ and $\rho_{1}^{\mathrm{eq}}$. For the present computation we set: $\lambda . \rho^{*}=10^{-6}$ and $\rho_{1}^{\mathrm{eq}}=\frac{1}{5}$. We obtain $\phi=-2.5$. For computational reasons we set $l=10 \tau^{*} U^{*}$ and we obtain $\delta_{1}=25$ and $\delta_{2}=250$.

Let us notice that the state equation $\sigma_{i}=\gamma\left(\rho_{0}^{\mathrm{s}}-\rho_{i}\right), i=1,2$, implies that $\rho_{1}^{\mathrm{eq}}<\rho_{2}^{\mathrm{eq}}=1$. (Recall: the index 2 refers to the solid-liquid interface, $\sigma_{2}=\sigma_{\mathrm{SL}}$ ). All the assumptions on data presented in the mathematical analysis section are satisfied.

The functions $\rho, \rho^{\prime}, \sigma_{\mathrm{SL}}$ and $\sigma_{\mathrm{SL}}^{\prime}$ obtained are presented below and in Fig. 1. We obtained: $\sigma_{\mathrm{SL}}\left(P_{\mathrm{C}}\right)=66.8, \sigma_{\mathrm{SL}}^{\prime}\left(P_{\mathrm{C}}\right)=1.01 \times 10^{6}, \theta_{\mathrm{d}}=103.9^{\circ}, \rho\left(P_{\mathrm{C}}\right)=2.51 \times 10^{-4},\left\|\rho^{\prime}\right\|_{\infty}=175.6$.

We note that the surface tension $\sigma_{\mathrm{SL}}$ and its gradient $\sigma_{\mathrm{SL}}^{\prime}$ are found from the values of $\rho, \rho^{\prime}$ and using the state equation $\sigma_{i}=\gamma\left(\rho_{0}^{\mathrm{s}}-\rho_{i}\right), i=1,2$. As a matter of fact, since $\sigma_{\mathrm{LG}}^{\mathrm{eq}}=\gamma\left(\rho_{0}^{\mathrm{s}}-\rho_{1}^{\mathrm{eq}}\right)$ and $\sigma_{\mathrm{SL}}^{\mathrm{eq}}=\gamma\left(\rho_{0}^{\mathrm{s}}-\rho^{*}\right)$, one can deduce the values of the constants $\gamma$ and $\rho_{0}^{\mathrm{s}}$. Then, using the state equation: $\sigma_{\mathrm{SL}}(y)=\gamma\left(\rho_{0}^{\mathrm{s}}-\rho(y)\right)$, we obtain the value of the surface tension coefficient $\sigma_{\mathrm{SL}}$. Finally, we have

$$
\theta_{\mathrm{d}}=\cos ^{-1}\left(\frac{\sigma_{\mathrm{SG}}^{\mathrm{eq}}-\sigma_{\mathrm{SL}}\left(P_{\mathrm{C}}\right)}{\sigma_{\mathrm{LG}}^{\mathrm{eq}}}\right) \text {, }
$$

35 where $P_{\mathrm{C}}$ denotes the triple point liquid-solid-gas.

The choice of the two parameters values of $\left(\lambda \rho^{*}\right)$ and $\rho_{1}^{\mathrm{eq}}$ is the main uncertainty of the model. The present choice leads to admissible surface tension coefficient $\sigma_{\mathrm{SL}}$ and dynamical wetting angle $\theta_{\mathrm{d}}$. 

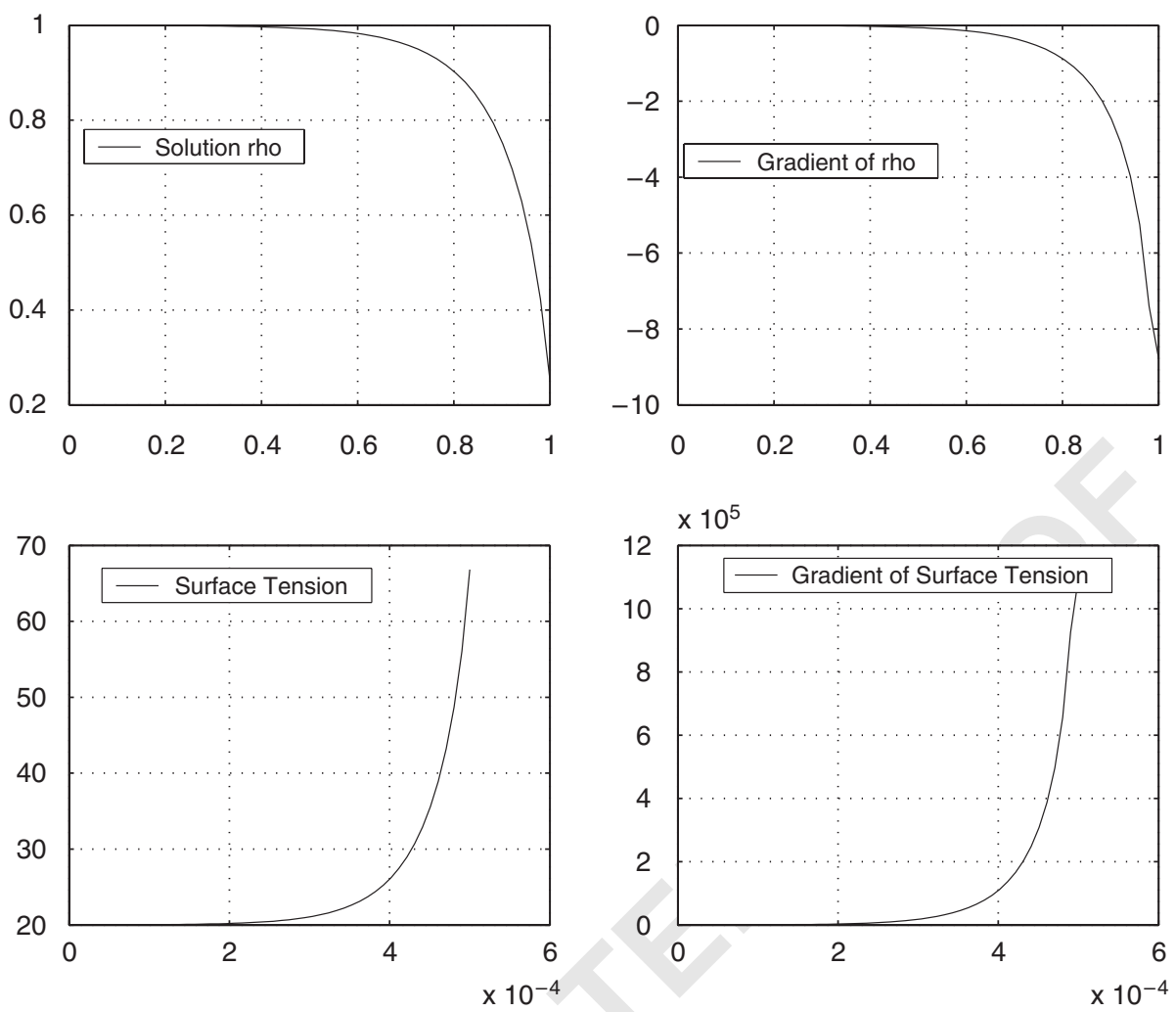

Fig. 1. From left to right: $\rho, \rho^{\prime}, \sigma_{\mathrm{SL}}, \sigma_{\mathrm{SL}}^{\prime}$.

\section{Influence of the local Marangoni term on the 2D bulk flow}

Let $\theta_{\mathrm{d}}, \sigma_{\mathrm{SL}}, \sigma_{\mathrm{SL}}^{\prime}$ and $\Gamma_{\mathrm{LG}}$ be given, we compute the solution of a simplified HFSM:

3 the Stokes model without curvature term $(\kappa=0)$. The goal is to observe numerically the influence of the local Marangoni source term $\sigma_{\mathrm{SL}}^{\prime}$ on the bulk fluid motion.

5 The simplified HFSM considered is the following. Given $\theta_{\mathrm{d}}, \sigma_{\mathrm{SL}}^{\prime}$ and $\Gamma_{\mathrm{LG}}$, find $(u, p)$ satisfying: $\partial_{1} \Sigma_{i 1}+\partial_{2} \Sigma_{i 2}=0, i=1,2$, and $\operatorname{div}(u)=0$ in $\Omega ; \Sigma_{n}=\Sigma_{\tau}=0$ on $\Gamma_{\mathrm{LG}} \cup \Gamma_{\text {out }}$;

$7 u=U_{\mathrm{S}}$ on $\Gamma_{\mathrm{SL}}^{\mathrm{M}}$;

$$
u \cdot n=0 \quad \text { and } \quad \Sigma_{\tau}=-\beta\left(u-U_{\mathrm{S}}\right)+\frac{1}{2} \sigma_{\mathrm{SL}}^{\prime} \quad \text { on } \Gamma_{\mathrm{SL}}^{\mathrm{m}} .
$$

9 The slip coefficient $\beta \approx \mu / h_{1} \approx 10^{-3} / 10^{-8}=10^{5}$ ( $h_{1}$ is the layer thickness) [7]. The only source terms of the model are $U_{\mathrm{S}}$ and $\sigma_{\mathrm{SL}}^{\prime}$. And, for $\sigma_{\mathrm{SL}}^{\prime} \equiv 0$, the unique solution is

$11(u, p)=\left(U_{\mathrm{S}}, 0\right)$ (the pressure being defined up to a constant).

We solve $\left(P_{\mathrm{ST}}\right)$ using the Hood-Taylor finite element method. The pressure equation is

13 solved using the augmented Lagrangian method and the Uzawa's algorithm. 

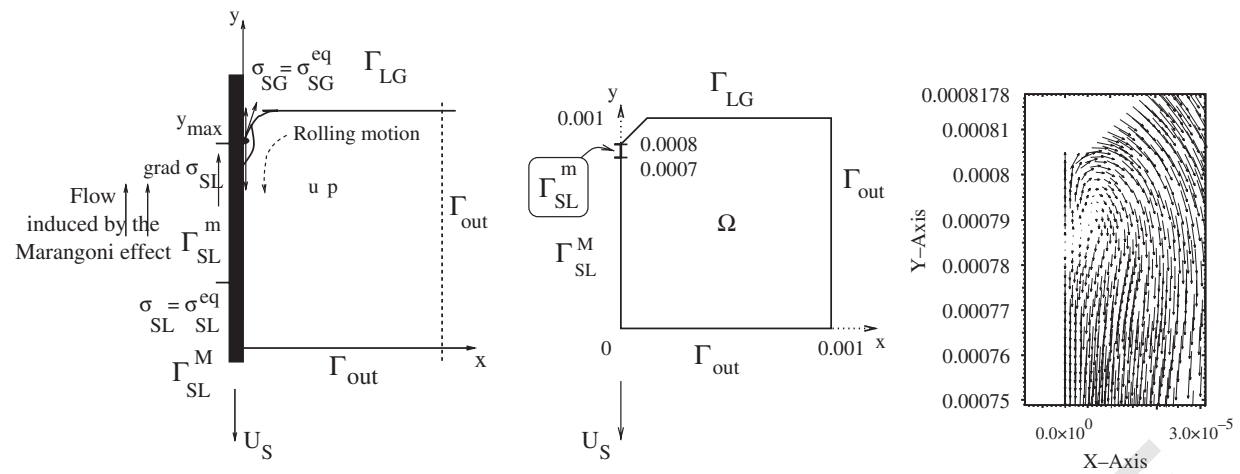

Fig. 2. Left. Plunging plate configuration. Middle. The Stokes case test. Right. Zoom on $\vec{u}$ near the contact point.

1 We set $U_{\mathrm{S}}=\left(0,-10^{-2}\right)^{\mathrm{T}}$ and $L=10^{-3}$ (in IS units). We set $\tau^{*}=10^{-3}$ hence $l \approx$ $\tau^{*} U^{*}=10^{-5}$ and $\varepsilon \approx l / L \approx 10^{-2}$. We have the Capillary number $C a=\mu U^{*} / \sigma_{\mathrm{SL}} \approx 10^{-6}$ 3 and the Reynolds number $R e=\hat{\rho} U^{*} L^{*} / \mu \approx 50$.

It remains to set the two following parameters: the slip coefficient $\beta$ and the given surface 5 tension gradient $\sigma_{\mathrm{SL}}^{\prime}$

For all tests, we set $\beta=10^{5}$, and $\sigma_{\mathrm{SL}}^{\prime}(y)=\sigma_{\max }^{\prime} \times \exp \left(\frac{y-y_{\mathrm{m}}}{y_{\mathrm{cp}}-y_{\mathrm{m}}}-1\right) \times\left(\frac{y-y_{\mathrm{m}}}{y_{\mathrm{cp}}-y_{\mathrm{m}}}\right)$ if

$7 y_{\mathrm{m}} \leqslant y \leqslant y_{\mathrm{cp}}$ and $\sigma_{\mathrm{SL}}^{\prime}(y)=0$ if not; where $y_{\mathrm{m}}=0.00075$ is the middle point of the boundary part $\Gamma_{\mathrm{SL}}^{\mathrm{m}}$ and $y_{\mathrm{cp}}=0.0008$ is the contact point $y$-coordinate, Fig. 2.

9 Therefore, the present given function $\sigma_{\mathrm{SL}}^{\prime}(y)$ behaves qualitatively similar to the computed one in previous section, Fig. 1.

11 First, we consider $\sigma_{\max }^{\prime}=10^{3}$. We observe a simple flow.

Second, we consider $\sigma_{\max }^{\prime}=5 \times 10^{3}$. We observe a more complex flow. The given source term $g_{\text {slip }}$ changes of sign in the vicinity of $7.8 \times 10^{-4}$. The computed $y$-coordinate velocity $u_{2}$ changes sign too, in the same area. Thus, we observe a local recirculation: the Marangoni term induces a recirculation in the vicinity of the contact line, see Fig. 2.

In conclusion, we would like to point that the model is too simplified to interpret these numerical results from the mechanical point of view. To this end, one must take into account the free surface dynamic, the capillary forces and eventually consider the local slip boundary conditions in the upper part of the vicinity of the triple line, i.e. on $\Gamma_{\mathrm{LG}}$. Nevertheless, these numerical results show clearly the effects of the local slip boundary condition on the fluid motion in the bulk. These numerical results are a first step for the simulation of the rolling motion and the dynamic of the contact angle using the model presented in $[7,1]$.

\section{References}

[1] T.D. Blake, et al., Experimental evidence of nonlocal hydrodynamic influence on the dynamic contact angle, Phys. Fluids 11 (8) (1999) 1995-2007.

[2] R.G. Cox, The dynamics of spreading of liquids on a solid surface. Part 1. Viscous flow, J. Fluid Mech. 168 (1986) 169-194. 
1 [3] V.E.B. Dussan, S.H. Davis, On the motion of a fluid-fluid interface along a solid surface, J. Fluid Mech. 65 (1974) 71-95.

3 [4] P.G. de Gennes, Wettings: statics and dynamics, Rev. Mod. Phys. 57 (1985) 3.

[5] D. Gilbarg, N. Trudinger, Elliptic Partial Differential Equations of Second Order, Springer, Berlin, 1977.

5 [6] L.M. Hocking, A moving fluid interface. Part II. The removal of the force singularity by a slip flow, J. Fluid Mech. 79 (1977) 209-229.

7 [7] Y.D. Shikhmurzaev, The moving contact line problem on a smooth solid surface, Int. J. Multiphase Flow 19 (4) (1993) 589-610. 